\title{
Mejora de los servicios públicos en el fortalecimento de la gestión municipal
}

\author{
Mg. Rafael Gaviria del Águila \\ rafaga43@hotmail.com \\ Escuela de posgrado \\ Universidad Cesar Vallejo \\ ORCID: 0000-0002-2745-1587 \\ José Manuel Delgado Bardales \\ jmdelgadob@ucvvirtual.edu.pe \\ Escuela de posgrado \\ Universidad César Vallejo \\ Tarapoto - Perú \\ ORCID: 0000-0001-6575-2759 \\ Scopus autor ID: 24070333700 \\ Código Renacyt: P0050554
}

\section{RESUMEN}

La investigación tuvo como objetivo conocer y proponer mejoras de los servicios públicos en la gestión municipal. El tipo de investigación es no experimental básica y el diseño de estudio es una revisión sistemática; las revisiones sistemáticas se realizaron de investigaciones científicas, cuya unidad de análisis se realizó en base a estudios originales primarios. En conclusión, los artículos revisados ante cambios en la administración pública, los servicios públicos, debe estar siempre orientados al ciudadano, cuyo fin debe ser la satisfacción y el cumplimiento de resultados de calidad, considerando los tipos y características del poblador y de las condiciones geográficas. Debe haber compromiso entre el ciudadano y la entidad, participación constante no sólo en la evaluación o fiscalización sino en la toma de decisiones. La prestación de los servicios públicos centrados en los ciudadanos es el desafío del gobierno nacional y local para resolver las necesidades de la población, asegurando la accesibilidad de los mismos, gestionando estándares de calidad y asegurando el apoyo político para el desarrollo social y humano.

Palabras clave: servicios públicos, gestión, municipio 


\title{
Improvement of public services in strengthening municipal management
}

\begin{abstract}
The objective of the investigation was to know and propose improvements to public services in municipal management. The type of research is basic non-experimental and the study design is a systematic review; The systematic reviews were carried out on scientific investigations, whose unit of analysis was based on original primary studies. In conclusion, the articles reviewed in the face of changes in public administration, public services, must always be oriented to the citizen, whose aim must be the satisfaction and fulfillment of quality results, considering the types and characteristics of the population and the geographical conditions. . There must be commitment between the citizen and the entity, constant participation not only in the evaluation or inspection but also in decision-making. The provision of public services centered on citizens is the challenge of the national and local government to meet the needs of the population, ensuring their accessibility, managing quality standards and ensuring political support for social and human development.
\end{abstract}

Keywords: public services, management, municipality

Artículo recibido: 03 nov. 2020 Aceptado para publicación: 07 dic. 2020 Correspondencia: rafaga43@ hotmail.com. Conflictos de Interés: Ninguna que declarar 


\section{INTRODUCCIÓN}

En la actualidad, la preocupación que se establece como parte del funcionamiento de las instituciones públicas, se asocia con la prestación de los servicios básicos, reconocidos como derechos fundamentales (Matías, 2014. p.317). En ese sentido los objetivos de la nueva gestión estatal es la de cambiar la gerencia pública de la misma forma que no sea una asociación, pero que sea más institucional. La administración pública, como proveedor de servicios para la población, le será difícil liberarse de las responsabilidades de proveer servicios eficaces y seguros entre la bolsa, además, no demostrara una orientación dirigida a la producción de ganancias, que es el compromiso imprescindible de una asociación que desea ubicarse en el mercado de una manera competitiva; que esto no sería un objetivo imposible de lograr, se ha demostrado con habilidades obtenidas en otros países (Holanda, Suecia, Estados Unidos, Gran Bretaña, Nueva Zelanda,etc.), que por muchos años han sido atados a transformaciones, que a la vez están teniendo mayor importancia en otros sitios del mundo, así como en América Latina (Schroeder, 2016. p. 8).

Asimismo, una sociedad compleja como la actual, con realidades propias en la que vivimos, con grupos sociales nuevos, con múltiples organizaciones no gubernamentales, con necesidades nuevas y problemas nuevos, una gestión con tintes burocráticos no reacciona adecuadamente a los intereses personales y colectivos. Una asociación estatal, así, padece de graves dificultades de adecuarse y de refutación placentera a las demandas de una población cada vez con mayor exigencia (Aguiar, 2016, p. 37). En tal sentido, los gobiernos locales sobre todo en el Perú, no son una excepción, como instituciones descentralizadas, dentro de sus competencias representan directamente a la barriada, fomentan la correcta facilitación de los servicios básicos, el crecimiento integral, armónico e integral de su circunscripción.

Además, el Estado peruano aprueba el DS No 004-2013 -PCM, donde la política nacional de innovación de la gestión estatal, dentro de los objetivos específicos, establece mejorar la gestión por procedimientos e impulsar la reducción administrativa en todos los entes públicos con el fin de obtener resultados alentadores en la mejoría de los procesos y servicios dirigidos a la población, cuyos ámbitos de aplicación corresponde a las entidades públicas. En ese contexto corresponde a las municipalidades establecer e implementar nuevos modelos de gestión pública, a fin de mejorar los servicios públicos a la población usuaria y no percibe el bajísimo servicio en la recolección de desperdicios, por las calles adyacentes a los mercados, asentamientos humanos, plazas y jardines de la ciudad de Yurimaguas. 
También, se observa que existe serios problemas relacionados a la prestación de los servicios públicos que ofrecen las entidades del Estado en sus diferentes órganos de gobierno, lo que repercute grandemente en la insatisfacción de los usuarios, a pesar de los esfuerzos que se viene realizando, existe desconfianza en la solución de los problemas sobre todo en limpieza pública y ornato, tránsito y seguridad vial, mercados y camal, entre otros servicios; lo que está ocasionando poca participación de la población. Uno de los servicios públicos que se evidencia de mayor malestar en los ciudadanos de la provincia de Alto Amazonas es la limpieza pública, el recojo de basura y el ornato público, a pesar de contar con herramientas como compactadoras, camiones recolectores, la falta de pago al personal, presupuesto, para algo tan vital como es mantener una ciudad limpia (Diario La Región, 2020); en ese sentido, es patente ver como la deficiente gestión de los servicios públicos genera descontento en la población, a tal punto de poner en riesgo la salud de la ciudadanía.

Por lo mencionado se formuló el siguiente problema: ¿Cómo es la mejora de los servicios públicos en la gestión municipal?, y como Objetivo: Conocer y proponer mejoras de los servicios públicos en la gestión municipal

\section{ESTRATEGIAS METODOLÓGICAS O MATERIALES Y MÉTODOS}

El tipo fue cualitativo y el diseño de estudio es una revisión sistemática, las revisiones sistemáticas son investigaciones científicas en las cuales la unidad de análisis son los estudios originales primarios, constituyen una herramienta esencial para sintetizar la información científica disponible, incrementar la validez de las conclusiones de estudios individuales e identificar áreas de incertidumbre donde sea necesario realizar investigación.

La recolección de datos se realizó a través de la revisión bibliográfica de artículos de investigaciones tanto nacionales como internacionales que tuvieron como tema principal la mejora de los servicios públicos y su repercusión en la buena atención del servicio y la satisfacción del ciudadano. Se estableció la búsqueda siempre y cuando se tuvo acceso al texto completo del artículo científico.

La técnica de análisis fue la revisión sistemática evaluando cada uno de los artículos para una comparación de los puntos o características en las cuales concuerda y los puntos en los que existe discrepancia entre artículos nacionales e internacionales. Además, de acuerdo a criterios técnicos pre establecidos, se realizó una evaluación crítica e intensiva de cada artículo, a partir de ello, se determinó la calidad de la evidencia y la fuerza de recomendación para cada artículo. 


\section{RESULTADOS Y DISCUSIÓN}

\section{RESULTADOS}

3.1. Tablas 1: Estudios revisados sobre eficacia de la restricción proteica en pacientes con insuficiencia renal crónica

\section{DATOS DE LA PUBLICACIÓN}

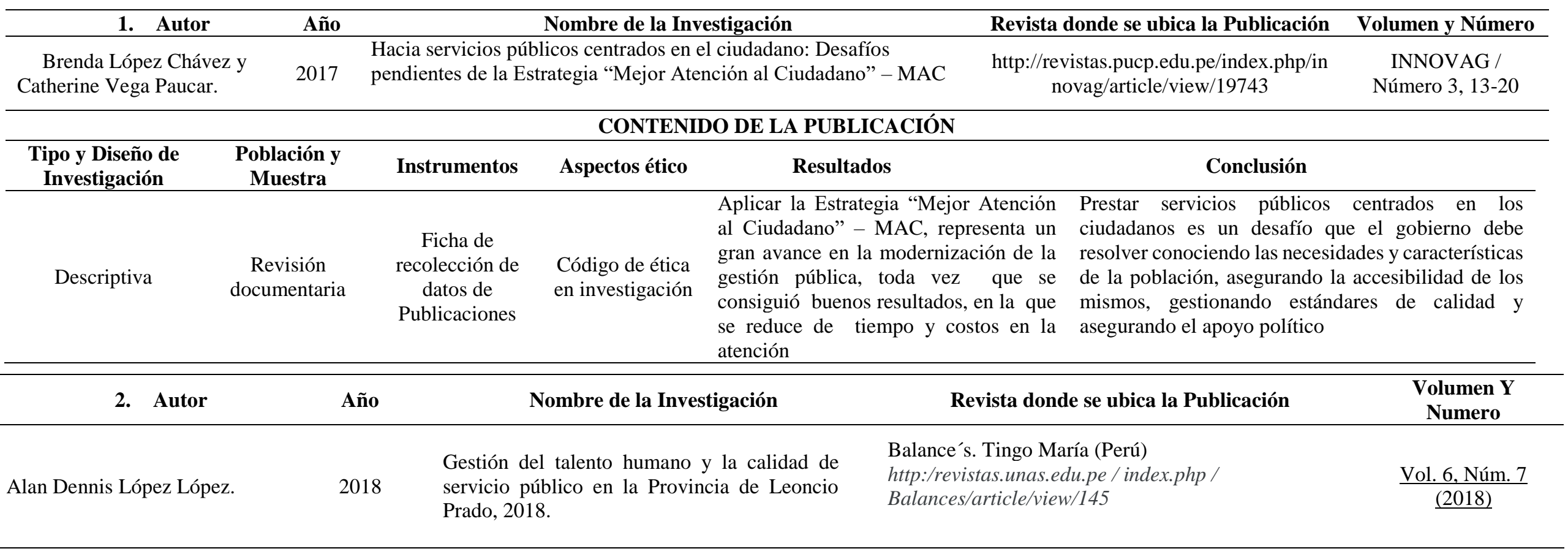

\section{CONTENIDO DE LA PUBLICACIÓN}

\begin{tabular}{|c|c|c|c|c|c|}
\hline $\begin{array}{l}\text { Tipo y Diseño de } \\
\text { Investigación }\end{array}$ & Población y Muestra & Instrumentos & Aspectos ético & Resultados & Conclusión \\
\hline $\begin{array}{l}\text { Descriptivo-correlacional } \\
\text { y diseño no experimental }\end{array}$ & $\begin{array}{l}180 \text { funcionarios y } \\
530 \text { usuarios de la } \\
\text { Municipalidad } \\
\text { Provincial de Leoncio } \\
\text { Prado }\end{array}$ & Cuestionarios & $\begin{array}{l}\text { Consentimiento } \\
\text { informado }\end{array}$ & $\begin{array}{l}\text { Los resultados muestran las } \\
\text { opiniones acerca de que el } \\
\text { conocimiento de los funcionarios } \\
\text { si es necesario para mejorar la }\end{array}$ & $\begin{array}{l}\text { Se determinó que la gestión del talento humano } \\
\text { se relaciona significativamente con la calidad de } \\
\text { servicio público, lo que indica que sí existe una } \\
\text { correlación positiva muy alta entre la gestión del } \\
\text { talento humano y la calidad de servicio público. }\end{array}$ \\
\hline
\end{tabular}




$\begin{array}{lr}\text { La muestra } & \text { se } \\ \text { determinó aplicando } \\ \text { la fórmula } & \text { para } \\ \text { poblaciones } & \text { finitas, } \\ \text { obteniéndose } & 282 \\ \text { usuarios. } & \end{array}$

La muestra se

determinó aplicando

poblaciones finitas,

usuarios. calidad de servicio en su área de trabajo.

Las habilidades y competencias laborales de los funcionarios si es necesario para mejorar la calidad de servicio en su área de trabajo
A si mismo se estable que el conocimiento, las habilidades y las actitudes de los funcionarios, nos permite demostrar, que existe una correlación positiva muy alta entre el conocimiento y la calidad de servicio público.
Aproximación al enfoque de la calidad de los servicios públicos: la carta de servicios https://dialnet.unirioja.es/servlet/articulo
Boletín de la Asociación Andaluza de

Bibliotecarios, ISSN 021 3-6333, Año No $31, N^{\circ}$ 112, 2016, págs. 30-56

\begin{tabular}{|c|c|c|c|c|c|}
\hline \multicolumn{6}{|c|}{ CONTENIDO DE LA PUBLICACIÓN } \\
\hline $\begin{array}{l}\text { Tipo y Diseño de } \\
\text { Investigación }\end{array}$ & $\begin{array}{l}\text { Población y } \\
\text { Muestra }\end{array}$ & Instrumentos & Aspectos ético & Resultados & Conclusión \\
\hline Descriptivo. & $\begin{array}{c}\text { Revisión } \\
\text { documentaria }\end{array}$ & $\begin{array}{l}\text { Ficha de } \\
\text { recolección de } \\
\text { datos de } \\
\text { Publicaciones }\end{array}$ & $\begin{array}{l}\text { Código de ética en } \\
\text { investigación }\end{array}$ & $\begin{array}{l}\text { Implantar una serie de } \\
\text { mecanismos } \\
\text { modernización enmarcados } \\
\text { en la dinámica del modelo } \\
\text { EVAM. El objetivo principal } \\
\text { del plan es conseguir que la } \\
\text { administración tenga un } \\
\text { enfoque al cliente, a su } \\
\text { personal y a la mejora de la } \\
\text { eficiencia en sus resultados. }\end{array}$ & $\begin{array}{l}\text { Si una organización se plantea poner en marcha una } \\
\text { herramienta que ayuda alcanzar la excelencia en la } \\
\text { prestación de sus servicios como es la Carta de } \\
\text { Servicios, debe contar con una adecuada } \\
\text { planificación estratégica, basados en su visión, } \\
\text { misión y valores. } \\
\text { La carta de servicio es una herramienta de } \\
\text { comunicación y transparencia entre la ciudadanía y la } \\
\text { administración, sobre la base de un acuerdo y } \\
\text { compromiso, indicadores y estándares de calidad, es } \\
\text { cambiante en el tiempo y camino a la excelencia. }\end{array}$ \\
\hline
\end{tabular}

Ciencia Latina Revista Científica Multidisciplinar, Ciudad de México, México. ISSN 2707-2207 / ISSN 2707-2215 (en línea), julio-diciembre, 2020, Volumen 4, Número 2. https://doi.org/10.37811/cl_rcm.v4i2.151 p. 1246 


\section{DATOS DE LA PUBLICACIÓN}

\begin{tabular}{|c|c|c|c|c|}
\hline 4. Autor & Año & Nombre de la Investigación & Revista donde se ubica la Publicación & \multirow{2}{*}{$\begin{array}{l}\text { Volumen Y Numero } \\
\text { Vol. 14, n. 1, p. } 144 \text { - } \\
\text { 158, jun. 2019. ISSN } \\
\text { 2631- }\end{array}$} \\
\hline Cedeño. J. y Jara. I & 2019 & $\begin{array}{l}\text { Los servicios públicos } \\
\text { domiciliarios en Ecuador. } \\
\text { Recopilación y análisis de } \\
\text { documentos relacionados }\end{array}$ & $\begin{array}{c}\text { Revista SATHIRI, sembrador } \\
\text { DOI https://doi.org/10.32645/13906925.724 }\end{array}$ & \\
\hline \multicolumn{5}{|c|}{ CONTENIDO DE LA PUBLICACIÓN } \\
\hline $\begin{array}{l}\text { Tipo y Diseño de } \\
\text { Investigación }\end{array}$ & $\begin{array}{c}\text { Población y } \\
\text { Muestra }\end{array}$ & $\begin{array}{c}\text { Aspectos } \\
\text { ético }\end{array}$ & Resultados & clusión \\
\hline $\begin{array}{l}\text { Cualitativa de tipo analítica- } \\
\text { descriptiva }\end{array}$ & $\begin{array}{l}\text { Revisión } \\
\text { documentaria, } \\
\text { repositorios } \\
\text { digitales. }\end{array}$ & $\begin{array}{l}\text { Consentimi } \\
\text { ento } \\
\text { informado. }\end{array}$ & $\begin{array}{ll}\text { Se evidencia que en Ecuador existe una } & \text { Ecuador a pe } \\
\text { extensa normativa en torno a la gestión } & \text { equidad, ase } \\
\text { de los servicios públicos domiciliarios; } & \text { manteniendo } \\
\text { sin embargo, en la ejecución de la } & \text { dicta su Con } \\
\text { prestación de los servicios de algunos } & \text { Se evidencia } \\
\text { de ellos aparecen desorganizaciones y } & \text { sean zonales } \\
\text { casos atípicos que vienen desde } & \text { buen servici } \\
\text { tiempos anteriores a la actual } & \\
\text { Constitución, sobre todo, por las } & \\
\text { concesiones y las características de las } & \\
\text { empresas públicas y las instituciones } & \\
\text { que las manejan. }\end{array}$ & $\begin{array}{l}\text { orar sus niveles de acceso, } \\
\text { y calidad del agua, sigue } \\
\text { ly por debajo de las que } \\
\text { rentes territorios del país, } \\
\text { s o locales carecen de un }\end{array}$ \\
\hline
\end{tabular}

\begin{tabular}{|c|c|c|c|c|c|}
\hline 5. & Autor & Año & Nombre de la Investigación & Revista donde se ubica la Publicación & Volumen Y Numero \\
\hline Rubio,L.C & & 2017 & $\begin{array}{l}\text { Servicios públicos } \\
\text { domiciliarios: responsabilidad } \\
\text { social y tercerización }\end{array}$ & $\begin{array}{c}\text { http://dx.doi.org/10.26564/21453381.720 } \\
\text { Colombia }\end{array}$ & Vol. 10. Numero 16 \\
\hline
\end{tabular}

\section{CONTENIDO DE LA PUBLICACIÓN}

\begin{tabular}{|c|c|c|c|c|c|}
\hline $\begin{array}{l}\text { Tipo y Diseño de } \\
\text { Investigación }\end{array}$ & $\begin{array}{c}\text { Población y } \\
\text { Muestra }\end{array}$ & Instrumentos & Aspectos ético & Resultados & Conclusión \\
\hline $\begin{array}{l}\text { Diseño descriptivo- } \\
\text { correlacional }\end{array}$ & & & $\begin{array}{l}\text { Consentimient } \\
\text { o Informado. }\end{array}$ & & $\begin{array}{l}\text { El sistema actual de servicio público domiciliario no es } \\
\text { sostenible en términos de responsabilidad social } \\
\text { empresarial se requiere grandes cambios, apoyado en el } \\
\text { sector privado por los siguientes factores: } \\
\text { Cobrar el metro cúbico muy caro a los usuarios } \\
\text { residenciales y comerciales hace menos competitivo el }\end{array}$ \\
\hline
\end{tabular}

Ciencia Latina Revista Científica Multidisciplinar, Ciudad de México, México.

ISSN 2707-2207 / ISSN 2707-2215 (en línea), julio-diciembre, 2020, Volumen 4, Número 2.

https://doi.org/10.37811/cl rem.v4i2.151 p. 1247 
sistema, lo cual conlleva implicaciones negativas en temas de incentivo al trabajo, desprotección a los recursos naturales y falta de motivación para implementar acciones de RSE.

\section{Autor}

Año

Nombre de la Investigación

La calidad percibida de los servi

Monsiváis. A cios públicos locales y la

confianza institucional en

México
Revista donde se ubica la Publicación

https://doi.org/10.22198/rys2019/31/1206
Volumen Y Numero

\section{ISSN e-2448-4849}

región y sociedad / año

31 / 2019 / e1206

ISSN e-2448-4849

región y sociedad / año

$31 / 2019$ / e1206

\section{CONTENIDO DE LA PUBLICACIÓN}

\begin{tabular}{|c|c|c|c|c|c|}
\hline $\begin{array}{l}\text { Tipo y Diseño de } \\
\text { Investigación }\end{array}$ & Población y Muestra & Instrumentos & Aspectos ético & Resultados & Conclusión \\
\hline Descriptivo & $\begin{array}{l}\text { Su población objetivo } \\
\text { son las personas de } 18 \\
\text { años en adelante, } \\
\text { residentes en hogares de } \\
\text { ciudades de } 100000 \text { o } \\
\text { más habitantes en las } 32 \\
\text { entidades del país de } \\
\text { México. }\end{array}$ & $\begin{array}{l}\text { Recolección de datos a } \\
\text { través de Encueta }\end{array}$ & & $\begin{array}{l}\text { Los resultados constatan que } \\
\text { la poca confianza política que } \\
\text { se observa en este país está } \\
\text { asociada con la opinión que } \\
\text { tiene la ciudadanía del } \\
\text { funcionamiento del sistema } \\
\text { político. También se muestra } \\
\text { que las experiencias } \\
\text { cotidianas y concretas de las } \\
\text { personas con el desempeño } \\
\text { de los gobiernos locales son } \\
\text { decisivas para moldear su } \\
\text { confianza en las instituciones } \\
\text { políticas. }\end{array}$ & $\begin{array}{l}\text { La confianza o desconfianza política de } \\
\text { los ciudadanos está ligada estrechamente } \\
\text { con la calidad de los bienes públicos de } \\
\text { su entorno inmediato. } \\
\text { Vale la pena insistir en que los hallazgos } \\
\text { contribuyen a mostrar que calidad de los } \\
\text { bienes públicos locales son } \\
\text { determinantes para que la ciudadanía } \\
\text { perciba que las democracias hacen una } \\
\text { diferencia en su calidad de vida. }\end{array}$ \\
\hline
\end{tabular}

Ciencia Latina Revista Científica Multidisciplinar, Ciudad de México, México.

ISSN 2707-2207 / ISSN 2707-2215 (en línea), julio-diciembre, 2020, Volumen 4, Número 2.

https://doi.org/10.37811/cl_rcm.v4i2.151 p. 1248 


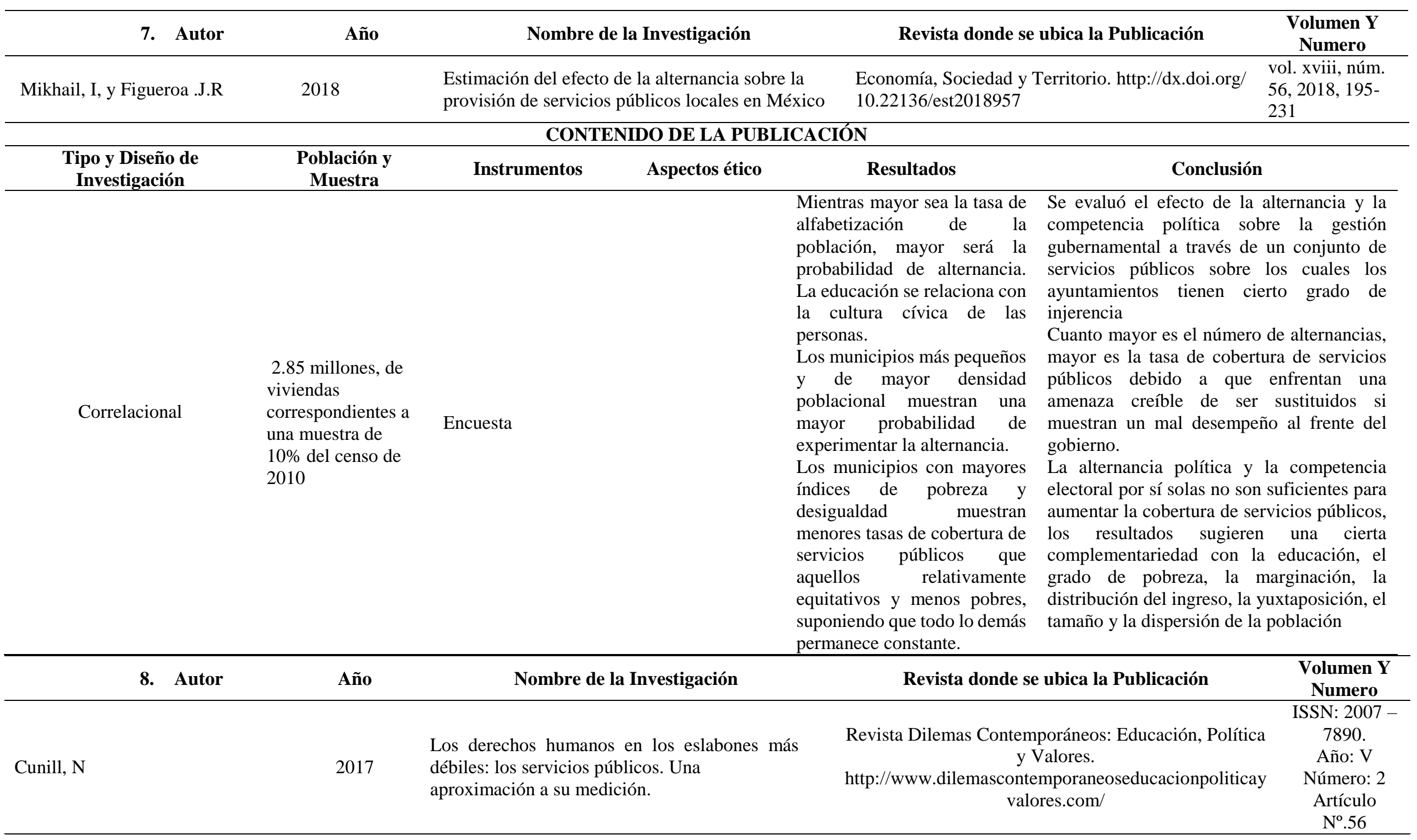

Ciencia Latina Revista Científica Multidisciplinar, Ciudad de México, México. ISSN 2707-2207 / ISSN 2707-2215 (en línea), julio-diciembre, 2020, Volumen 4, Número 2. https://doi.org/10.37811/cl rem.v4i2.151 p. 1249 


\section{CONTENIDO DE LA PUBLICACIÓN}

\begin{tabular}{|c|c|c|c|c|c|}
\hline $\begin{array}{l}\text { Tipo y Diseño de } \\
\text { Investigación }\end{array}$ & $\begin{array}{l}\text { Población } \\
\text { y Muestra }\end{array}$ & Instrumentos & Aspectos ético & Resultados & Conclusión \\
\hline Descriptiva & $\begin{array}{l}800 \\
\text { personas }\end{array}$ & Cuestionario & & $\begin{array}{l}\text { Este trabajo pretende contribuir a esta } \\
\text { discusión ofreciendo una primera batería de } \\
\text { indicadores que puedan usarse para cualquier } \\
\text { servicio social e idealmente para todos ellos, a } \\
\text { fin de no solo poner en práctica una mirada } \\
\text { integral, sino de proveer medidas que puedan } \\
\text { dar señales de alerta oportunas y también ser } \\
\text { usadas por la propia ciudadanía y las } \\
\text { organizaciones de la sociedad civil defensoras } \\
\text { de derechos. }\end{array}$ & $\begin{array}{l}\text { El desafío que cabe enfrentar y del } \\
\text { que este trabajo intenta ser un primer } \\
\text { paso, consiste en acercar los } \\
\text { derechos a los servicios públicos, } \\
\text { lugares donde efectivamente se } \\
\text { zanja su realización, y por ende, el } \\
\text { enfrentamiento de parte significativa } \\
\text { de las desigualdades sociales. }\end{array}$ \\
\hline
\end{tabular}

\begin{tabular}{|c|c|c|c|c|c|c|}
\hline 9. Autor & Año & \multicolumn{3}{|c|}{ Nombre de la Investigación } & Revista donde se ubica la Publicación & $\begin{array}{c}\text { Volumen Y } \\
\text { Numero }\end{array}$ \\
\hline \multirow[t]{2}{*}{ VERGARA. J.C, y Maza, F.J } & 2017 & \multicolumn{2}{|c|}{$\begin{array}{l}\text { Valoración de los servicios públicos domiciliarios de } \\
\text { Cartagena de Indias Colombia. }\end{array}$} & $\begin{array}{l}\text { rev.fac.cienc.ec } \\
\text { http://dx.doi.ors }\end{array}$ & $\begin{array}{l}\text { rev.fac.cienc.econ } \\
\text { http://dx.doi.org/10.18359/rfce. } 2656\end{array}$ & $\begin{array}{l}\text { Vol. XXV } \\
\text { (1), Junio } \\
\text { 2017, 95-103 }\end{array}$ \\
\hline & \multicolumn{6}{|c|}{ CONTENIDO DE LA PUBLICACIÓN } \\
\hline $\begin{array}{l}\text { Tipo y Diseño de } \\
\text { Investigación }\end{array}$ & $\begin{array}{l}\text { Población y } \\
\text { Muestra }\end{array}$ & Instrumentos & Aspectos ético & Resultados & Conclusió & \\
\hline Correlacional & $\begin{array}{l}250 \text { familias de las } \\
15 \text { comunas de } \\
\text { Cartagena } \\
\text { Indias. }\end{array}$ & Cuestionario & & $\begin{array}{l}\text { Los resultados sugieren dos } \\
\text { correlaciones fuertes entre las } \\
\text { percepciones de calidad y las } \\
\text { satisfacción con los servicios } \\
\text { domiciliarios, y las percepciones y } \\
\text { la satisfacción general, esta última } \\
\text { relacionada con la valoración de } \\
\text { todos los servicios públicos } \\
\text { recibidos -domiciliarios, salud, } \\
\text { educación, seguridad, } \\
\text { infraestructura y buen gobierno }\end{array}$ & $\begin{array}{l}\text { Las percepciones de cali } \\
\text { fuerte influencia con la sa } \\
\text { los servicios públicos dor } \\
\text { menor medida, con la satis } \\
\text { sobre los servicios públi } \\
\text { su vez se revela una relac } \\
\text { percepciones de calidad y } \\
\text { Por otra parte, con este est } \\
\text { a demostrar una relación e } \\
\text { encuestado y las percepci } \\
\text { debido a que, existe cierta } \\
\text { en las problemáticas que } \\
\text { los estratos socioeconómi } \\
\text { poca oferta de empresas } \\
\text { servicio, sólo conviven ol }\end{array}$ & $\begin{array}{l}\text { ad tienen una } \\
\text { facción sobre } \\
\text { ciliarios y, en } \\
\text { acción general } \\
\text { s recibidos, a } \\
\text { in fuerte entre } \\
\text { atisfacción. } \\
\text { dio no se llegó } \\
\text { re el perfil del } \\
\text { es de calidad, } \\
\text { omogeneidad } \\
\text { fectan a todos } \\
\text { s, sumado a la } \\
\text { roveedoras de } \\
\text { opolios. }\end{array}$ \\
\hline
\end{tabular}

Ciencia Latina Revista Científica Multidisciplinar, Ciudad de México, México. ISSN 2707-2207 / ISSN 2707-2215 (en línea), julio-diciembre, 2020, Volumen 4, Número 2. https://doi.org/10.37811/cl rem.v4i2.151 p. 1250 


\section{DATOS DE LA PUBLICACIÓN}

\begin{tabular}{|c|c|c|c|c|c|c|c|}
\hline 10. Autor & Año & \multicolumn{3}{|c|}{ Nombre de la Investigación } & \multicolumn{2}{|c|}{ Revista donde se ubica la Publicación } & $\begin{array}{l}\text { Volumen Y } \\
\text { Numero }\end{array}$ \\
\hline Córdova, J. y Ponce, A. & 2016 & \multicolumn{3}{|c|}{$\begin{array}{l}\text { Los tipos de corrupción y la satisfacción con } \\
\text { los servicios públicos. Evidencia del caso } \\
\text { mexicano. }\end{array}$} & $\begin{array}{l}\text { Región y sociedad } \\
\text { versión impresa ISSN 1870-3925 } \\
\text { https://dx.doi.org/10.22198/rys.201 }\end{array}$ & .70 .9344 & $\begin{array}{l}\text { vol.29, } \mathrm{N}^{\mathrm{o}} .70 \\
\text { pp.231-262. }\end{array}$ \\
\hline \multicolumn{8}{|c|}{ CONTENIDO DE LA PUBLICACIÓN } \\
\hline $\begin{array}{l}\text { Tipo y Diseño de } \\
\text { Investigación }\end{array}$ & $\begin{array}{l}\text { Población y } \\
\text { Muestra }\end{array}$ & Instrumentos & $\begin{array}{l}\text { Aspectos } \\
\text { ético }\end{array}$ & \multicolumn{2}{|r|}{ Resultados } & \multicolumn{2}{|c|}{ Conclusión } \\
\hline Correlacional & $\begin{array}{c}28960 \\
\text { encuestados }\end{array}$ & Encuestas & $\begin{array}{l}\text { Consentim } \\
\text { iento } \\
\text { informado. }\end{array}$ & \multicolumn{2}{|c|}{$\begin{array}{l}\text { Acerca del efecto de la corrupción sobre la } \\
\text { satisfacción de los usuarios es que: la percepción de } \\
\text { corrupción reduce la satisfacción con la provisión de } \\
\text { los servicios públicos. } \\
\text { La percepción de la grande y la pequeña corrupción } \\
\text { reduce el grado de satisfacción con cada servicio } \\
\text { urbano provisto por el municipio }\end{array}$} & \multicolumn{2}{|c|}{$\begin{array}{l}\text { La percepción de la gran corrupción } \\
\text { resulta ser la más dañina, cuando se } \\
\text { explican las caídas en la satisfacción } \\
\text { con los servicios } \\
\text { Es necesaria una estrategia que } \\
\text { controle y disminuya gradualmente } \\
\text { las múltiples dimensiones de } \\
\text { corrupción. } \\
\text { Para ello se requiere la revisión de } \\
\text { manuales y reglamentos, el fomento } \\
\text { de políticas de trasparencia, el } \\
\text { fortalecimiento de valores éticos en la } \\
\text { administración pública y de los } \\
\text { procedimientos de control y reglas } \\
\text { informales que norman los incentivos } \\
\text { de los burócratas, para que decidan ya } \\
\text { no incurrir en actos corruptos. }\end{array}$} \\
\hline 11. Autor & Año & \multicolumn{3}{|c|}{ Nombre de la Investigación } & \multicolumn{2}{|c|}{ Revista donde se ubica la Publicación } & $\begin{array}{l}\text { Volumen Y } \\
\text { Numero }\end{array}$ \\
\hline Villa, Cando, Alcoser y Ramos & 2017 & $\begin{array}{l}\text { Estudio de los se } \\
\text { de Riobamba } \\
\text { usuarios. }\end{array}$ & $\begin{array}{l}\text { 'icios público } \\
\text { la satisfac }\end{array}$ & $\begin{array}{l}\text { en la ciudad } \\
\text { ón de los }\end{array}$ & $\begin{array}{l}\text { DOI: } \\
\text { http://dx.doi.org/10.17993/3cemp.20 }\end{array}$ & $17.060432 .55-71$ & $\begin{array}{l}\text { 3C Empresa } \\
\text { (Edición núm. 32) } \\
\text { Vol.6- } \mathrm{N}^{\circ} 4 \\
\text { Noviembre'17 - } \\
\text { febrero '18, } 55 \text { - } \\
71\end{array}$ \\
\hline
\end{tabular}

Ciencia Latina Revista Científica Multidisciplinar, Ciudad de México, México.

ISSN 2707-2207 / ISSN 2707-2215 (en línea), julio-diciembre, 2020, Volumen 4, Número 2.

https://doi.org/10.37811/cl rem.v4i2.151 p. 1251 


\section{CONTENIDO DE LA PUBLICACIÓN}

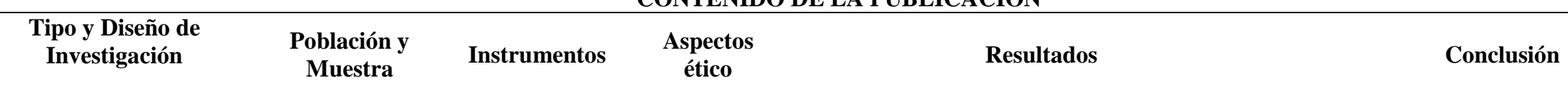

\begin{tabular}{|c|c|c|c|c|c|}
\hline $\begin{array}{l}\text { Tipo transversal, con } \\
\text { enfoque cualitativo }\end{array}$ & $\begin{array}{l}2.378 \text { personas } \\
\text { de la ciudad de } \\
\text { Riobamba }\end{array}$ & $\begin{array}{l}\text { Cuestionario } \\
\text { Entrevistas }\end{array}$ & $\begin{array}{l}\text { Código de } \\
\text { ética de } \\
\text { investigaci } \\
\text { ón }\end{array}$ & $\begin{array}{l}\text { Los ciudadanos perciben que la mayoría de los } \\
\text { servicios públicos que prestan las entidades } \\
\text { gubernamentales son muy satisfactorias. Del mismo } \\
\text { modo manifiestas que los servicios públicos más } \\
\text { utilizados son en primer lugar los servicios de salud } \\
\text { pública, seguido de las carreteras y obras públicas y } \\
\text { la educación pública superior. Sin embargo, la } \\
\text { percepción de la atención se ha delimitado en dos } \\
\text { aspectos: positivos y negativos: dentro de los ítems } \\
\text { positivos los más relevantes en la actualidad son: Las } \\
\text { muy cómodas instalaciones que tienen las entidades } \\
\text { públicas, los horarios oportunos y la modalidad de } \\
\text { turnos electrónicos para la atención. Con relación a } \\
\text { los items negativos se destacan: La lentitud en los } \\
\text { trámites, las filas muy largas y que todavía existen } \\
\text { funcionarios groseros. } \\
\text { También los ciudadanos piensan que la atención en } \\
\text { las instituciones públicas en los últimos años ha } \\
\text { mejorado considerablemente y en bajo porcentaje de } \\
\text { ciudadanos cree que ha empeorado la atención. }\end{array}$ & $\begin{array}{l}\text { La mayoría de ciudadanos de } \\
\text { Riobamba tienen una buena } \\
\text { percepción de los servicios públicos } \\
\text { evaluándolos como muy satisfactorios } \\
\text { y que los servicios públicos más } \\
\text { utilizados son: Salud, carreteras, obras } \\
\text { públicas y educación superior. Así } \\
\text { mismo entre los aspectos que la } \\
\text { ciudadanía considera que han } \\
\text { mejorado están: La infraestructura, } \\
\text { instalaciones muy cómodas, horarios } \\
\text { adecuados y turnos electrónicos. Sin } \\
\text { embargo, persisten aspectos negativos } \\
\text { como: Lentitud en los trámites, filas } \\
\text { muy largas y todavía existen } \\
\text { funcionarios groseros. } \\
\text { Finalmente, el ciudadano debe poner } \\
\text { de su parte para que la ejecución de las } \\
\text { políticas permita una integración que } \\
\text { promueva la calidad del servicio y la } \\
\text { satisfacción de los usuarios. }\end{array}$ \\
\hline
\end{tabular}




\section{DISCUSIÓN}

Culminada el análisis sistemático de los artículos científicos, del total revisado, el $46 \%$ corresponden a México y España, el 64\% a Perú, Colombia, Ecuador y Chile. El tipo de investigación es descriptiva con sus diferentes variantes.

La mayor parte de las conclusiones de los autores, coinciden en que una buena administración de los servicios públicos, debe estar centrado en las necesidades del ciudadano y que a la vez se refleje en la satisfacción de la misma.

Sin embargo, López, B y Vega, C. sostiene que, prestar servicios públicos centrados en los ciudadanos es un desafío que el gobierno debe resolver conociendo las necesidades y características de la población, asegurando la accesibilidad de los mismos, gestionando estándares de calidad y asegurando el apoyo político

Además, López, A. Determinó que la gestión del talento humano se relaciona significativamente con la calidad de servicio público, lo que indica que sí existe una correlación positiva muy alta entre la gestión del talento humano y la calidad de servicio público. A si mismo se estable que el conocimiento, las habilidades y las actitudes de los funcionarios, nos permite demostrar, que existe una correlación positiva muy alta entre el conocimiento y la calidad de servicio público.

Asimismo, Vergara, J y Maza, F. Concluyen que las percepciones de calidad tienen una fuerte influencia con la satisfacción sobre los servicios públicos domiciliarios y, en menor medida, con la satisfacción general sobre los servicios públicos recibidos, a su vez se revela una relación fuerte entre percepciones de calidad y satisfacción.

Igualmente, Córdova, J. y Ponce, A. Manifiestan que la percepción de la gran corrupción resulta ser la más dañina, cuando se explican las caídas en la satisfacción con los servicios. Por lo mencionado se hace necesaria una estrategia que controle y disminuya gradualmente las múltiples dimensiones de corrupción. Para ello se requiere la revisión de manuales y reglamentos, el fomento de políticas de trasparencia, el fortalecimiento de valores éticos en la administración pública y de los procedimientos de control y reglas informales que norman los incentivos de los burócratas, para que decidan ya no incurrir en actos corruptos.

También Villa, Cando, Alcoser y Ramos. La mayoría de ciudadanos de Riobamba tienen una buena percepción de los servicios públicos evaluándolos como muy satisfactorios y que los servicios públicos más utilizados son: Salud, carreteras, obras públicas y educación superior. 
Así mismo entre los aspectos que la ciudadanía considera que han mejorado están: La infraestructura, instalaciones muy cómodas, horarios adecuados y turnos electrónicos. Sin embargo, persisten aspectos negativos como: Lentitud en los trámites, filas muy largas y todavía existen funcionarios groseros. Finalmente, el ciudadano debe poner de su parte para que la ejecución de las políticas permita una integración que promueva la calidad del servicio y la satisfacción de los usuarios.

\section{CONCLUSIÓN O CONSIDERACIONES FINALES}

Los 11 artículos revisados concluyen que, ante los diferentes cambios que se generan en la administración pública, los servicios públicos, debe estar siempre orientados al ciudadano, cuyo fin debe ser la satisfacción y el cumplimiento de resultados de calidad, considerando los tipos y características del poblador y de las condiciones geográficas de la misma.

Debe haber un pacto y compromiso entre el ciudadano y la entidad, participación constante no solo en la evaluación o fiscalización sino en la toma de decisiones.

La prestación de los servicios públicos centrados en los ciudadanos es el desafío del gobierno nacional y local para resolver las necesidades de la población, asegurando la accesibilidad de los mismos, gestionando estándares de calidad y asegurando el apoyo político para el desarrollo social y humano.

Se recomienda generar políticas públicas de transparencia y de una adecuada comunicación para generar una confianza en el manejo eficiente y eficaz de los recursos financieros.

\section{LISTA DE REFERENCIAS}

Cedeño. J. y Jara. I. (2019). Los servicios públicos domiciliarios en Ecuador. Recopilación y análisis de documentos relacionados https://doi.org/10.32645/13906925.724

Córdova, J. y Ponce, A. (2016). Los tipos de corrupción y la satisfacción con los servicios públicos. Evidencia del caso mexicano. https://dx.doi.org/10.22198/rys.2017.70.a344

Cunill, N. (2018). Los derechos humanos en los eslabones más débiles: los servicios públicos. Una aproximación a medición http://www.dilemascontemporaneoseducacionpoliticayvalores.com/

Fernández, J. (2016). Aproximación al enfoque de la calidad de los servicios públicos: la carta de servicios.http://.dialnet.unirioja.es /servlet /articulo 
López B., y Vega, C. (2017). Hacia servicios públicos centrados en el ciudadano: Desafíos pendientes de la Estrategia "Mejor Atención al Ciudadano" - MAC. InnovaG, (3), 13-20. Recuperado a partir de http://revistas.pucp.edu.pe/index.php/innovag/article/view/19743

López, A. (2108). Gestión del talento humano y la calidad de servicio público en la Provincia de Leoncio Prado, 2018.- Perú. http:/revistas.unas.edu.pe / index.php / Balances/article/view/145

Monsiváis, A. (2019). La calidad percibida de los servicios públicos locales y la confianza institucional en México. Región Y Sociedad, 31, e1206. https://doi.org/10.22198/rys2019/31/1206

Rubio, L. (2017). Servicios públicos domiciliarios: responsabilidad social y tercerización Colombia. DOI: http://dx.doi.org/10.26564/21453381.720

Soto, I, y Figueroa, J. (2018). Estimación del efecto de la alternancia sobre la provisión de servicios públicos locales en México. Economía, sociedad y territorio, 18(56), 195231. https://dx.doi.org/10.22136/est2018957

Vergara, J. y Maza, F. (2017). Valoración de los servicios públicos domiciliarios de Cartagena de Indias Colombia. http://dx.doi.org/10.18359/rfce.2656.

Villa, H.V., Cando, A.R., Alcoser, F.E. y Ramos, R.A. (2017). Estudio de los servicios públicos en la ciudad de Riobamba y la satisfacción de los usuarios. 3C Empresa, investigación y pensamiento crítico, 6(4), 55-71. DOI: http://dx.doi.org/10.17993/3cemp.2017.060432.55-71 